\title{
Uma Proposta Para Análise de Dados Abertos Utilizando A Linguagem R
}

João Pedro Albino - jp.albino@unesp.br ${ }^{1}$

Resumo - Os dados abertos governamentais podem se constituir em um recurso crítico para as estratégias das micro, pequenas e médias empresas (PME). Para obter novos e valiosos insights nesses dados, a linguagem de código aberto R pode ser utilizada para agregar valor aos negócios das PMEs por meio da análise de dados. A proposta deste trabalho é demonstrar, de forma simplificada, a construção de algoritmos flexíveis na linguagem $\mathrm{R}$, realizando uma análise exploratória com referência às PMEs nas bases públicas do BNDES. Como metodologia, utilizou-se o processo de ciência de dados com o intuito que este exemplo aplicado seja reproduzível em alguns setores tendo como base os dados abertos governamentais.

Palavras-chave: Análise de Dados; Dados Abertos; Linguagem R; PME; Ciência de Dados.

\section{A Proposal For Open Data Analysis Using The R Language}

\begin{abstract}
Open government data can be a critical resource for strategies proposals on micro, small and medium-sized enterprises (SMEs). On other hand, to gain valuable new insights on data, $\mathrm{R}$, an open source language, can be used and add value to SME businesses through data analysis. The proposal of this work is to demonstrate, in a simplified way, the construction of flexible algorithms in the language $\mathrm{R}$, carrying out an exploratory analysis with reference to SMEs in public BNDES bases. As a methodology, the data science process was used in order to make this applied example, reproducible in some sectors based on open government data.
\end{abstract}

Keywords: Data Analysis; Open Data; Language R; SMEs; Data Science. 


\section{Introdução}

Com a massiva quantidade de dados disponíveis nas mais variadas formas e plataformas, além da proliferação de tecnologias voltadas para Internet, as micro, pequenas e médias empresas (PMEs), apesar de suas restrições de tamanho, receita e pequeno número de funcionários, podem também se beneficiar desta vasta informação disponível e gerar valor para si próprias (PREEZ, 20I4).

Pequenas e médias empresas representam a maioria das empresas e são responsáveis por ofertar grande parcela do emprego total no setor privado na maioria das economias (BECK, 2007).

A contratação de funcionários em empresas com até 250 empregados constitui mais de $60 \%$ do emprego total na indústria transformadora em muitos países, o que justifica a afirmação de que "as PMEs formam o emergente setor privado nos países pobres e, assim, formam a base para o crescimento liderado pelo setor privado (LEME, 20I5).

Pesquisas realizadas em vários países, no entanto, também mostram que as pequenas e médias empresas são mais limitadas no seu funcionamento e crescimento do que as grandes empresas e que o acesso a serviços financeiros representa a fatia mais importante entre estas restrições (PREEZ, 20I4).

À medida que a economia do Brasil cresce, crescem também as oportunidades para os empresários de PMEs. O país tem mais de I2 milhões de negócios, dos quais 8,3 milhões são mico empreendedores individuais (MEI). Os pequenos negócios também respondem por 52,2\% dos empregos gerados pelas empresas no país. No Brasil, o Banco Nacional de Desenvolvimento Econômico e Social (BNDES) define como PMEs empresas que possuem receita operacional anual de US\$ 38 milhões ou menos (LEME, 20I5).

Os esforços políticos destinados às PMEs têm sido muitas vezes justificados pelos bancos de fomento de desenvolvimento porque as pequenas e médias empresas são o motor de inovação e crescimento e ajudam a reduzir a pobreza, uma vez que são organizações que empregam os maiores recursos em força de trabalho. Entretanto, afirma Beck (2007), as PMEs são limitadas por falhas institucionais e de mercado.

Os principais fatores e tendências para as PME brasileiras, no entender de Leme (2015), podem ser resumidos como: as PME contribuem em $20 \%$ do PIB do Brasil; mais de $50 \%$ dos empregos formais do Brasil estão nas PME; no Brasil, a tecnologia tem papel crescente nas PME; e o empreendedorismo é uma escolha para o crescimento, mais que uma necessidade nessas organizações.

Sendo assim, mais e mais as PMEs compreendem o valor da análise de dados para as suas estratégias em gerar valor aos seus clientes. Pesquisas como as realizadas em Preez (20I4), Leme (20I5) e Rijmenam (20I5) demonstraram que as empresas que utilizam análise de dados saem-se melhor que suas concorrentes. A vantagem de segmentação das PMEs, é tal 
que na maioria das vezes as empresas não estão restritas e dependentes de uma infraestrutura de Tecnologia da Informação e Comunicação (TIC) ultrapassada e possuem diretrizes menos rigorosas. As pequenas e médias empresas podem mudar e experimentar novas soluções mais facilmente (BECK, 2007).

Como a quantidade de dados disponíveis atualmente na Economia da Informação - um campo de estudos interdisciplinar entre Economia, Ciência da informação e Comunicação que trata da informação como mercadoria e bem de produção necessária às atividades econômicas - numerosos órgãos públicos e privados buscam aumentar a transparência dos processos e do seu desempenho ao publicar dados relevantes on-line e compartilhá-los com o público (THE ECONOMIST, 20IO).

De acordo com Ubaldi (2013), os dados de governos, disponibilizados em conjuntos legíveis e vinculados podem ser pesquisados e manipulados utilizando-se de ferramentas de busca (ou pesquisa) padrão. Isto se constitui em um novo recurso crítico para abastecer mudanças na criação de valor aos seus diferentes usuários (econômicos, sociais e políticos). A questão econômica e social dos dados abertos está se estabelecendo com evidências cada vez mais sólidas em muitas iniciativas nacionais e locais, de acordo com as iniciativas de Dados Abertos Governamentais da Organização para a Cooperação e Desenvolvimento Econômico (OCDE).

Nesta perspectiva, para a OCDE a criação de valor potencial também é esperada em termos políticos e sociais. Portanto ao lidar com a criação de valor é essencial identificar quais os principais beneficiários deste valor que está sendo criado. Para que os dados se beneficiem do mesmo grau de inovação e dinamismo que caracterizam a utilização por entidades privadas da Internet, é fundamental se repensar o papel dos provedores de dados e perceber que apenas disponibilizando dados brutos não é condição suficiente para se gerar valor, conclui Ubaldi (20I3).

Existem muitas oportunidades para as PMEs obterem conhecimentos e ideias valiosas a partir de seus dados proprietários já existentes ou no uso de novos conjuntos de dados. As pequenas e médias empresas têm que pensar em soluções inovadoras para ver oportunidades nos dados internos, bem como de fora de sua organização, assegura Preez (20I4). Portanto, uma vez que isto se torne também um fator importante para as PMEs e se estas pequenas corporações estiverem dispostas a investir tempo e dinheiro em uma estratégia de uso de análise de dados para agregar valor ao seu negócio, tais empresas também podem alcançar resultados consideráveis e superar os seus concorrentes, completa Beck (2007).

Para ajudar em sua estratégia de uso dessa grande quantidade de dados acessíveis (big data), as PMEs podem utilizar softwares de código aberto (open-source). Diante disso, organizações que antes dependiam de caras plataformas proprietárias e legadas para análise estatística estão adotando uma nova alternativa, a plataforma de código aberto gratuita denominada " $R$ " (SMITH, 20I5).

1 A Economia da Informação assumiu grande importância pela publicação do artigo de GREENWALD, Bruce C.; STIGLITZ, Joseph E. Externalities in Economies with Imperfect Information and Incomplete Markets, The Quarterly Journal of Economics, v. 101, n. 2, p. 229-264, 1986. Disponível em: https://doi.org/10.2307/1891114. Acesso em: 15 jun. 2016. 
Para obter novas e importantes perspectivas (insights) dos dados existentes ou externos, a linguagem $\mathrm{R}$ pode ser utilizada para análise, previsão e mineração de dados. Em grande parte, devido à sua natureza de código aberto, o ambiente de linguagem e programação $\mathrm{R}$ foi adotado em diversas universidades e empresas, atraídos pela sua constante atualização, mantidas pela equipe central de desenvolvimento da linguagem ou por terceiros (R CORE TEAM, 20I3).

Considerando as questões apresentadas anteriormente, a proposta deste trabalho é o de demonstrar, em uma abordagem simplificada, a construção de um exemplo aplicado no ambiente de linguagem e programação $\mathrm{R}$, realizando uma análise exploratória junto as bases de dados públicos do Banco Nacional de Desenvolvimento Econômico e Social (BNDES) com referência às PMEs. Será utilizado o modelo básico de processo de ciência de dados com o intuito de que seja reproduzível em alguns setores com base nos dados abertos governamentais.

\section{CONTEXTUALIZAÇÃO}

Pesquisa realizada pelo SAS Institute (2013) mostrou que 71\% das organizações não possuem uma estratégia de análise de dados. A grande maioria das organizações ainda não começou testar ou mesmo a planejar uma estratégia para trabalhar com grandes quantidades de dados, denominados na literatura como "big data" (BIG DATA, [s.d.]). De acordo com a pesquisa, 76\% dos altos executivos das PMEs entrevistados visualizam a utilização de análise de dados como uma oportunidade para uso e melhoria na gestão de suas empresas.

Saber trabalhar com grandes quantidades de dados procedentes de diversas localidades e com diferentes formatos é uma das habilidades mais desejadas atualmente, sustentam Isotani e Bittencourt (20I5). Isso ocorre devido ao crescimento exponencial dos dados gerados pela sociedade e à necessidade de minerar informações obtidas por meio da análise das conexões semânticas entre conceitos e relações presentes nestes dados.

O conceito de big data, segundo Rijmenam (20I5), não comporta apenas a área de conhecimento que estuda como tratar, analisar e obter informações a partir de conjuntos de dados de grande volume, variedade e velocidade e nem deve ser direcionado apenas para grandes organizações. Existem amplas oportunidades para as PME obterem novas perspectivas e conhecimentos importantes a partir de seus dados existentes ou novos conjuntos de dados.

Pelo lado das TICs, técnicas de aprendizado de máquina (machine learning) estão se tornando comuns no mundo dos negócios e de pesquisas para antecipar possibilidades de vendas e gestão de clientes, ou para ajudar aos pesquisadores compreender e obter insights sobre eventos futuros. (DEJAEGER et al., 20I2).

Segundo Simon (20I3), a aprendizagem de máquina é um subcampo da ciência da computação que evoluiu a partir do estudo do reconhecimento de padrões e da teoria da aprendizagem computacional em inteligência artificial. A aprendizagem automática explora o estudo e construção de algoritmos que podem aprender e fazer previsões sobre os dados. Tais algoritmos operam através da construção de um modelo de um conjunto exemplo de treinamento de observações de entrada, a fim de fazer previsões baseadas em dados ou decisões expressas como 
saídas, ao invés de seguir de forma estritamente estática as instruções do programa.

Definido como "análise preditiva", a técnica de aprendizagem automática, de acordo com Simon (20I3), se refere ao processo de coleta e processamento de dados e a aplicação posterior de alguma forma de análise matemática nesses dados para obter informações estratégicas. Com a automatização dos esforços de coleta de dados, cada vez mais dados estão sendo capturados, tornando a tarefa de extrair padrões interessantes cada vez mais desafiadora.

Embora a abordagem de aprendizagem de máquina requeira pessoal especializado, atualmente não exige custo muito elevado. De acordo com Mitchell et al. (20I2), muitas ferramentas de código aberto são gratuitas e o custo do hardware padrão se torna cada vez mais barato. O código-fonte aberto é uma parte importante da Web 3.0, porque se encaixa em um dos principais fatores na sua promoção: a colaboração.

A Web 3.0 representa a terceira geração da Internet, afirma Markoff (2006). Nesta nova geração os conteúdos online são organizados de forma semântica e muito mais personalizados para cada usuário: os sites, aplicações inteligentes e publicidade são baseados nas pesquisas e nos comportamentos dos internautas. Desta forma, o movimento open source (código aberto) obteve na Web 3.0 um ambiente seguro para a sua consolidação assim como também reforçou a necessidade para atender a reformulação maciça dos códigos-fonte de computação (IWASAKI, 2008).

Os softwares de código aberto baseiam-se no compartilhamento de informações, permitindo o uso de tecnologias sem a cobrança de licenças de software, o oposto do que é feito pelo sistema proprietário, o que inovou o mercado das TICs, declara Iwasaki (2008). Esse movimento de softwares com código aberto conta com o apoio e participação de comunidades compostas por pessoas espalhadas por todo o mundo.

O princípio fundamental do desenvolvimento de software de código aberto é o da produção pelos pares, no qual produtos como o código-fonte, diagramas e documentação ficam disponíveis para o público, sem nenhum custo. O movimento começou como uma resposta às limitações impostas pelo uso de código proprietário, e, desde então, tem se espalhado por diferentes campos (IWASAKI, 2008).

A possibilidade de inovação incremental a partir de processos colaborativos de uso da tecnologia permite mudanças disruptivas como a nova Economia da Informação compartilhada fundamentada nos aplicativos sociais e reaproveitamento de códigos e dados. Pessoas e organizações aptas a utilizar essas tecnologias abertas, que podem produzir novos negócios e novas soluções para a sociedade e a disponibilização de dados têm um potencial enorme de prover maior transparência e melhor participação dos cidadãos nas soluções dos seus problemas. As tecnologias abertas têm se revelado instrumentos alavancadores de inovação (ISOTANI e BITTENCOURT, 20I5). 
Dados que possam ser livremente utilizados, reutilizados e redistribuídos por qualquer pessoa ou organização, sujeitos, no máximo, à exigência de atribuição à fonte original e ao compartilhamento pelas mesmas licenças em que as informações foram apresentadas, estão em consonância com à lógica adotada nas comunidades de software de código aberto, concluem Isotani e Bittencourt (20I5).

Outra revolução tecnológica, nascida em 2009, o movimento de dados abertos, ou Open Data em inglês, tem objetivos semelhantes aos de outros movimentos "abertos", tais como o "código aberto", de "conteúdo aberto" e "acesso livre". Segundo a definição da Open Knowledge Foundation $^{2}$, os dados são considerados abertos quando qualquer pessoa pode acessar, usar, modificar e compartilhar livremente para qualquer finalidade (sujeito a, no máximo, a requisitos que preservem a proveniência e a sua abertura). Isso geralmente é satisfeito pela publicação dos dados em formato aberto e sob uma licença aberta (GOVERNO DIGITAL, [s.d.])

Já o conceito de dados abertos governamentais pode ser definido como uma metodologia para a publicação de dados do governo em formatos reutilizáveis, visando o aumento da transparência e maior participação política por parte do cidadão, além de gerar diversas aplicações desenvolvidas colaborativamente pela sociedade (GOVERNO DIGITAL, 20I5).

Dados abertos governamentais podem ser utilizados para ajudar as organizações e os cidadãos a entender melhor as políticas públicas e quão bem a administração executa suas estratégias e torná-lo responsável por ilegalidades ou resultados não alcançados. Isto é particularmente verdadeiro, pois uma quantidade considerável destes dados de governo estão se tornando progressivamente mais acessíveis e podem ser usados agregados a informações de outras fontes (por exemplo, informações proprietárias) para iniciativas de fiscalização da população, afirmam Ribeiro e Almeida (20II).

Por outro lado, dos governos espera-se que sejam capazes de oferecer uma ampla gama de dados acessíveis para incentivar a tomada de decisões baseadas em proeminências. Ubaldi (2013) considera que os dados abertos governamentais também devem ser vistos como uma fonte importante para fomentar o crescimento econômico, gerar novas formas de empreendimentos e capacitar a inovação social.

Sob este ponto de vista, os dados são um ativo importante e as informações que o governo detém os tornam um ativo extremamente valioso, afirma Manocha (20II). Assim, começa-se a revelar o valor destes dados quando se combinam diferentes fontes de dados relevantes. A transparência nos dados governamentais é uma característica importante, mas o valor real dos dados só pode ser alcançado se os mesmos forem explorados corretamente. Quando se começa a analisar os dados e utilizá-los para a inovação, otimização, previsão e predição, pode-se começar a evoluir em termos de informações transparentes, conclui Manocha (20II).

2 A Open Knowledge, antes conhecida como Open Knowledge Foundation, é uma organização sem fins lucrativos que promove conhecimento livre. Foi fundada em maio de 2004, em Cambridge. Disponível em: https://pt.wikipedia.org/wiki/Open_Knowledge. Acesso em: 08 jul. 2015. 
No caso do governo brasileiro, a ideia básica para dados abertos governamentais é tornar disponível um grande número de bases dados oficiais, cobrindo todos os aspectos da atividade governamental, permitindo que além do acesso aos dados, o público em geral possa compartilhá-los, fazendo o uso de processamento automatizado em computadores, conforme suas necessidades de informação (RIBEIRO e ALMEIDA, 20II; GOVERNO DIGITAL, 20I5).

Como pode ser visto, os dados abertos e os softwares de código aberto são vistos como recursos essenciais que podem ajudar as empresas a criar produtos e serviços novos e inovadores. Sob esta condição pesquisadores, formuladores de políticas e especialistas técnicos comemoraram o potencial da inovação de dados abertos para as empresas: estas podem acessar dados praticamente sem nenhum custo, obter conjuntos de dados novos que anteriormente não estavam disponíveis e criar produtos e serviços disruptivos. Discute-se também, continua o autor, que as PMEs estão particularmente bem posicionadas para se beneficiar da inovação de dados e códigos abertos.

A conjunção de dados abertos governamentais e os movimentos de softwares de código aberto estimularam o aparecimento de metodologias para a detecção de padrões e obtenção de novos insights para apoiar tomadas de decisão mais precisas. Entretanto, esta maior disponibilidade de dados e a premência das tecnologias abertas se tornaram demasiado complexas para os tradicionais sistemas automatizados proprietários, especialmente em um mundo onde as preocupações com o sigilo são fundamentais, declara Smith (20I5).

Como resultado, as empresas que antes dependiam de plataformas legadas proprietárias agora estão adotando uma nova alternativa que se configura como crucial para o desenvolvimento, criação e gestão de inovações e novos produtos, para isto utilizando conceitos de big data e open source, denominada ciência de dados. (CAVIQUE, 20I4).

\section{O PROCESSO DE CIÊNCIA DE DADOS}

Ciência de dados pode ser definida como um conjunto de práticas recomendadas usadas em empresas de tecnologia, em uma ampla gama de problemas que podem ser resolvidos utilizando-se dados, definem Schutt e O’Neil (20I3). E, de acordo com Cavique (20I4), é uma disciplina que estabelece relações entre diferentes áreas ou ramos de conhecimento dedicadas ao estudo e a análise de dados estruturados e não-estruturados, visando a extração de conhecimento.

Portanto, a ciência de dados permite transformar em projeto qualquer problema do mundo real e depois convertê-lo novamente em uma solução prática através de um processo que pode ser definido em seis (6) etapas básicas, como as mostradas na Figura I. 
Figura I. Processo de Ciência de Dados.

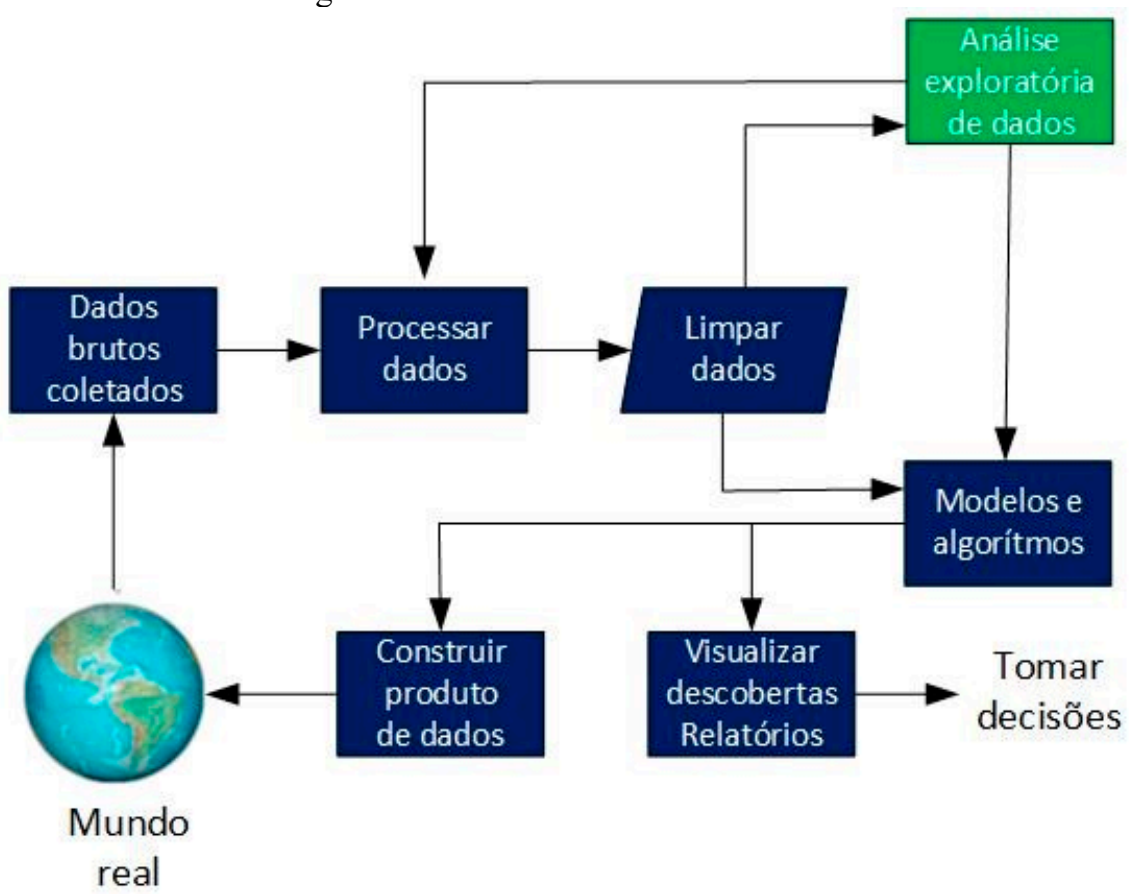

Fonte: Schutt e O’Neil (2013).

A primeira etapa importante antes de resolver um problema é definir exatamente qual é o problema (do Mundo Real) que se busca resolver. No caso de um processo de ciência de dados é preciso ser capaz de traduzir as questões sobre os dados em algo capaz de ser realizado ou praticado. (Schutt e O’Neil, 20I3).

No caso especificamente deste trabalho, busca-se resolver um problema de interesse das MPEs, buscando determinar quais seriam os possíveis desembolsos de investimentos do BNDES nas micro e pequenas empresas para no ano seguinte neste setor. Os dados dos anos considerados neste artigo, 2006 a 2015, encontram-se disponíveis no sítio do BNDES33. O principal problema a ser resolvido é determinar se o BNDES continuará a investir nesses segmentos nos mesmos patamares anteriores e com isto planejar a solicitação de recursos ao banco para investimentos em estratégias das MPEs.

Esta é uma etapa de descoberta e envolve a aquisição de dados de as fontes internas e externas identificadas, o que ajuda a responder à pergunta inicial. $\mathrm{O}$ importante no final desta primeira etapa, de acordo com Schutt e O’Neil (20I3), é se obter todas as informações e o contexto necessários para se resolver o problema e encontrar maneiras de obtê-las, quer se trate de consultar bancos de dados internos, ou a aquisição de conjuntos de dados externos.

3 BNDES. Evolução dos desembolsos. Disponível em: https://www.bndes.gov.br/wps/portal/ site/home/transparencia/estatisticas-desempenho/desembolsos. Acesso em: 11 dez. 2015. 
O próximo passo é a realização de uma análise exploratória dados (AED). A análise exploratória de dados é o primeiro passo a ser dado em direção à construção de um modelo para a análise dos dados. A AED é uma parte crítica do processo de ciência de dados, e também representa uma filosofia ou modo de fazer cálculos estatísticos praticados por uma estirpe de estatísticos provenientes da tradição do Bell Labs ${ }^{\circledR}$, afirmam Schutt e O’Neil (20I3).

A etapa seguinte à análise exploratória é o da construção do modelo. No nosso exemplo, queremos criar um modelo preditivo utilizando os dados dos últimos anos de desembolsos do BNDES para pequenas e médias empresas de todas as regiões do Brasil, e em todas as áreas de negócios. Nesta etapa, será construído um modelo de regressão para tentar prever valores do desembolso baseados em outros atributos dos valores (anos anteriores).

Usaremos o modelo de regressão linear múltipla. A abordagem de modelagem de regressão linear geralmente consiste em definir uma variável de resposta, a qual queremos prever e diversas variáveis de entrada. O modelo também assume que existe uma relação linear entre as variáveis de entrada e variável definida como resposta.

Após a construção e testes, o modelo final é entregue juntamente com os relatórios e gráficos elaborados. Também são entregues o código resultante e os documentos técnicos produzidos no desenvolvimento da solução modelada. De acordo com Schutt e O'Neil (20I3), nesta fase de operacionalização, após testes completos o modelo é implantado em um ambiente de produção em tempo real.

$\mathrm{Na}$ derradeira fase do processo de ciência de dados as principais conclusões são comunicadas a todas as partes interessadas (stackholders). Esta é considerada a parte mais desafiadora do processo: transformar os resultados obtidos e apresentá-los de uma forma que faça sentido e possa ser facilmente transmitida (CAVIQUE, 20I4).

Dentre os vários ambientes que oferecem a possibilidade de apoiar todas as etapas do processo de ciência de dados, a plataforma $\mathrm{R}$ vem se tornando, de forma crescente, um padrão de fato, assevera Smith (20I5). Possuindo recursos para mostrar elementos visuais como tabelas, gráficos e mapas, as ferramentas disponíveis no ecossistema $\mathrm{R}$ para visualização de dados fornecem uma maneira acessível para inferir e perceber tendências, discrepâncias e padrões nos dados.

\section{Ambiente de Programação R}

$\mathrm{O}$ "R" é um ambiente e uma linguagem para realizar cálculos estatísticos e visualização de gráficos, derivada da linguagem $\mathrm{S}^{\circledR}$, originalmente desenvolvida nos laboratórios da Bell Labs $^{\circledR}$. É software open source (de código aberto) para análise de dados que tem o apoio de uma comunidade colaborativa numerosa e ativa em todo o mundo. (The R Project, 20I5).

Desde a década de I990, o impulso vindo da academia e da indústria levou a linguagem de programação $\mathrm{R}$ a se tornar a ferramenta mais importante para aplicações em estatística computacional, visualização e ciência dados, afirma Kabacoff (20I5). 
Em todo o mundo, milhões de dados estatísticos e cientistas de dados utilizam o ambiente e a linguagem de programação $\mathrm{R}$ para resolver seus problemas mais desafiadores em áreas que compreendem desde biologia computacional até pesquisa de mercado quantitativa. $\mathrm{O}$ $\mathrm{R}$ se tornou a linguagem mais popular para a ciência de dados e uma ferramenta essencial para empresas voltadas para a análise, tais como Google ${ }^{\circledR}$, Facebook ${ }^{\circledR}$ e LinkedIn ${ }^{\circledR}$ (KABACOFF, 2015 e SMITH, 20I5).

De acordo com Diakopoulos e Cass (20I5), no âmbito das Io linguagens de programação mais utilizadas mundialmente, a linguagem $\mathrm{R}$ está bem posicionada. Na tabela na Figura 2, que compara o desempenho das linguagens no ano de 20I5, das seis principais linguagens utilizadas pelas organizações, a linguagem $\mathrm{R}$ é a que demonstra maior crescimento. Como se pode observar na classificação, o grande salto nas pontuações foi o da linguagem $R$, pois, no ano de 20I4, a linguagem estava em nono lugar e seu movimento reflete a crescente importância do big data para muitas especialidades. Apesar de ser voltada para uma área mais específica do que as outras relacionadas, a linguagem $\mathrm{R}$ tem mantido a sua popularidade nos últimos anos em razão da quantidade cada vez maior de dados disponíveis, concluem Diakopoulos e Cass (2015).

Figura 2. Linguagens mais utilizadas em TI.

\begin{tabular}{|c|c|c|c|}
\hline Language Rank & Types & Spectrum Ranking & Spectrum Ranking \\
\hline 1. Java & $\notin \square \square$ & 100.0 & -100.0 \\
\hline 2. $\mathrm{C}$ & a & 99.9 & -99.3 \\
\hline 3. $\mathrm{C}++$ & 05 & 99.4 & -95.5 \\
\hline 4. Python & \# $\square$ & 96.5 & -93.5 \\
\hline 5. C\# & $\notin \square \square$ & 91.3 & -92.4 \\
\hline 6. $\mathrm{R}$ & $\Psi$ & 84.8 & 84.8 \\
\hline 7. $\mathrm{PHP}$ & \# & 84.5 & 84.5 \\
\hline 8. JavaScript & $\notin \square$ & 83.0 & -78.9 \\
\hline 9. Ruby & $\notin \square$ & 76.2 & -74.3 \\
\hline 10. Matlab & $\square$ & 72.4 & -72.8 \\
\hline
\end{tabular}

Fonte: Diakopoulos e Cass (2015).

A linguagem atualmente é mantida pela "R Foundation", uma organização sem fins lucrativos que trabalha no interesse público. A Fundação foi criada pelos membros da equipe principal (core team) de desenvolvimento de projetos em R, com o objetivo de fornecer suporte técnico ao "Projeto R" e outras inovações em computação estatística (The R Project, 2015).

Desde a sua primeira versão em I999 até a sua versão atual (3.6), a linguagem amadureceu e o desenvolvimento e manutenção de novas implementações é garantido pela Fundação $\mathrm{R}$, que se propõe a centralizar os principais aprimoramentos da plataforma. Outro objetivo da 
Fundação é o de oferecer um ponto de referência para os indivíduos, instituições ou organizações que queiram apoiar ou interagir com a comunidade de desenvolvimento da linguagem, além de manter e administrar os direitos autorais do software e sua documentação. Além de apoiar o desenvolvimento continuado da linguagem R, a Fundação também se compromete a explorar, desenvolver e aplicar novas metodologias no ensino e treinamento em computação estatística e a organizar reuniões e conferências voltadas para a comunidade R (The R Project, 20I5).

O R é uma parte oficial do projeto GNU da Free Software Foundation e da Fundação $\mathrm{R}$ e tem objetivos semelhantes aos outros fundamentos de software de fonte aberta como a Fundação Apache ${ }^{\circledR}$ ou a Fundação GNOME ${ }^{\circledR}$.

\section{Por que utilizar o $R$}

Atualmente existem diversos pacotes estatísticos e gráficos disponíveis no mercado e concorrentes da linguagem R, tais como Microsoft Excel ${ }^{\circledR}$, SAS $^{\circledR}$, IBM SPSS $^{\circledR}$, Stata $^{\circledR}$ e Minitab $^{\circledR}$ (DIAKOPOULOS e CASS, 20I5). Portanto, qual seria a justificativa para se utilizar o ambiente R?

De acordo com Kabacoff (20I5), a linguagem R tem muitas características que recomendam seu uso:

$\sqrt{ }$ A maioria das plataformas de software estatísticos comerciais são caras;

$\sqrt{ }$ É uma plataforma estatística abrangente, oferecendo diversos tipos de técnicas analíticas;

$\sqrt{ }$ Contém rotinas estatísticas avançadas ainda não disponíveis em outros pacotes e tem atualizações constantes;

$\sqrt{ }$ Oferece recursos gráficos para a visualização de dados complexos;

$\sqrt{ }$ Sua funcionalidade pode ser integrada em aplicações escritas em outras linguagens.

$\sqrt{ }$ Pode ser executado em uma grande variedade de plataformas, incluindo Windows, Unix, Linux e Mac OSx.

A linguagem de programação R também se mostra uma ferramenta importante para se desenvolver análise e aprendizado de máquina, afirma Krill (20I5). Outra grande vantagem, segundo o autor, é a capacidade de se criar, analisar e distribuir código de alta qualidade e reprodutível na linguagem. Por ser estruturada como blocos de construção (building blocks), a maioria dos programas escritos em R são coleções de scripts organizadas em projetos, o que facilita sua implementação e manutenção.

A linguagem $\mathrm{R}$ possui um grande número de pacotes e bibliotecas prontas para uso. Tais recursos formam o ecossistema de pacotes em $\mathrm{R}$ ( $\mathrm{R}$ package ecosystem) mantidos por uma comunidade de estatísticos acadêmicos que possui muitos usuários ativos. $\mathrm{O}$ grupo de usuários 
LinkedIn $R$, por exemplo, possui mais de 30.000 membros, afirma Krill (20I5), e sites como o Meetup oferecem mais de 2800 grupos sobre o tema. A linguagem oferece ainda uma grande quantidade de documentação publicamente disponível para os usuários online.

Apesar de todos os seus benefícios, o $\mathrm{R}$ apresenta algumas deficiências. O gerenciamento de memória, a velocidade e a eficiência são, provavelmente, os maiores desafios, relata Krill (20I5). Além disso, pessoas que vêm de outros ambientes de programação podem também considerar a linguagem singular.

Entretanto, por sua característica de software de código aberto e suas constantes atualizações de versões, muitos avanços têm sido - e ainda estão sendo - realizados para trazer progressos nas deficiências existentes, conclui Krill (20I5).

\section{Exemplo Aplicado}

Para mostrar um exemplo simples do processo de uso da linguagem $\mathrm{R}$, utilizando dados de plataforma aberta, tomaremos como modelo básico o processo de ciência de dados definido em Schutt e O’Neil (20I3) e representado na Figura I.

No exemplo específico, foram obtidos os dados no formato do Microsoft Excel ${ }^{\circledR}$ (.xlsx). A Figura 3 mostra o trecho do código fonte em $\mathrm{R}$ para a carga do arquivo no ambiente da linguagem.

Figura 3. Código fonte em R para a carga do arquivo.

\# dowload, leitura e preparação do dataset

require(gdata)

require(rJava)

require(xlsx)

cnae<-read.xIs("Int2_1D_a_setorCNAE_MPME.xIsx", header=TRUE, row.names=1)

Após a carga dos dados brutos, é necessário avaliá-los antes de qualquer análise. Muitas vezes, os dados podem estar bastante confusos, especialmente se não foram coletados e armazenados com cuidado, contendo erros que poderão corromper a análise, tais como: valores definidos como nulos embora sejam realmente zero; valores duplicados; e valores ausentes (missing values). Cabe ao cientista de dados avaliar e verificar para se certificar de que os dados possuem informações precisas.

O próximo passo é a realização de uma análise exploratória dados. O código referente à análise exploratória em R é mostrado na Figura 4. 
$\operatorname{par}(\mathrm{m}$ frow $=\mathrm{c}(2,2))$

Figura 4. Código para análise exploratória inicial.

plot(log(cnae\$X2006:cnae\$X2015), main="Desembolsos BNDES de 2006 a 2015", xlab="Desembolsos em 2006", ylab="Desembolsos em 2015")

barplot(log(cnae\$X2015), xlab="Desembolsos em 2006", ylab="Desembolsos em 2015")

hist(log(cnae\$X2006:cnae\$X2015), main="Desembolsos BNDES de 2006 a 2015 ", xlab="Desembolsos 2006/2015") pie(abs(log(cnae\$X2015[1:10])), main="Desembolsos BNDES em 2015",

labels = c("Agropecuária", "Ind. extrat.", "Alimentos", "Bebidas", "Fumo", "Têxtil", "Vest. e acess.", "Couro",

"Madeira", "Papel"),

radius $=0.8$ )

Os gráficos iniciais da análise exploratória dos dados no exemplo estão representados nos gráficos e histograma da Figura 5 .

Figura 5. Análise exploratória dos dados.

Desembolsos BNDES de 2006 a 2015

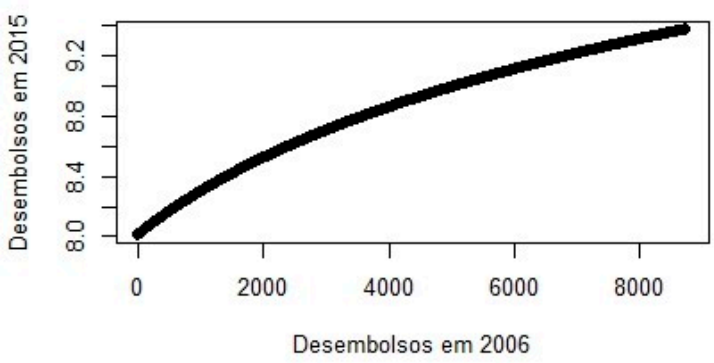

Desembolsos BNDES de 2006 a 2015

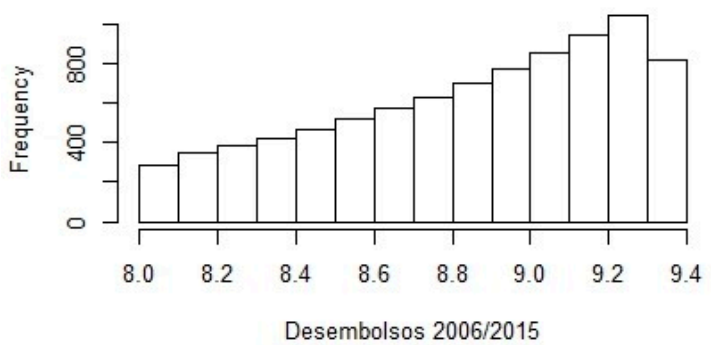

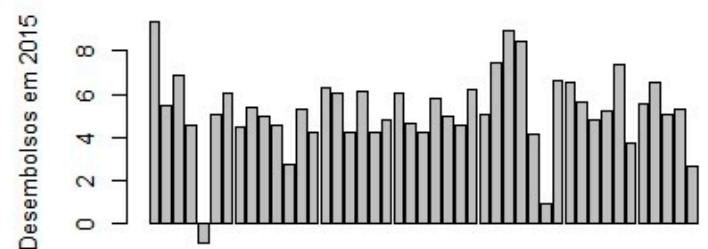

Desembolsos em 2006

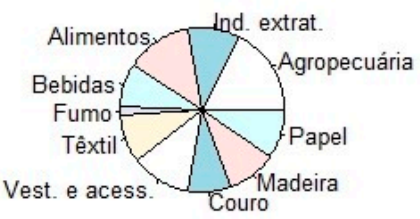

A etapa seguinte à análise exploratória é a construção do modelo. Neste exemplo aplicado, busca-se criar um modelo preditivo utilizando os dados de 2006 a 2015 com os desembolsos do BNDES para pequenas e médias empresas de todas as regiões do Brasil, e de todas as áreas de negócios.

Nesta etapa, será construído um modelo de regressão para tentar prever os futuros valores de desembolso baseados em outros atributos dos valores dos anos anteriores.

Será utilizado o modelo de regressão linear múltipla. A abordagem de modelagem de regressão linear geralmente consiste em uma variável de resposta, a qual se deseja predizer seu valor e diversas variáveis de entrada. Este modelo de regressão também assume que existe uma relação linear entre as variáveis de entrada e a variável de resposta. 
Na Figura 6, tem-se o código em R mostrando a construção de um modelo de regressão linear e a consequente impressão do resumo.

Figura 6. Construção do modelo em R.

\section{\# construção do modelo initial model $<-\operatorname{Im}(X 2015 \sim$. -Tipo, data $=$ cnae $)$ summary(initial model)}

Na Figura 7 podemos ver os detalhes do modelo utilizando o comando summary (resumo) da linguagem $\mathrm{R}$ na variável initial_model, o que dá informações detalhadas sobre o modelo e os coeficientes das variáveis em diferentes métricas.

Figura 7. Resumo do modelo.

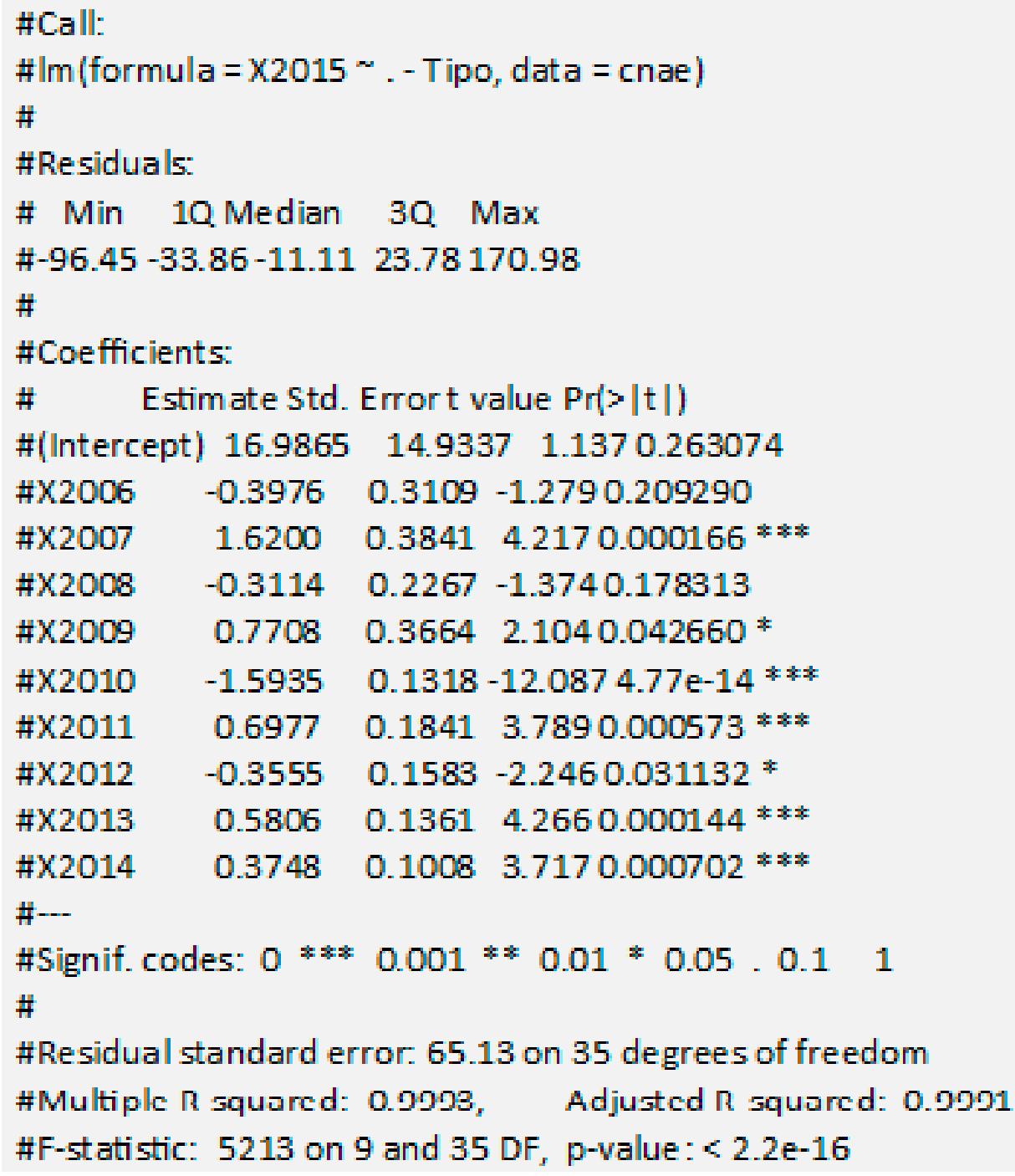


Observa-se que o valor de $\mathrm{R}^{2}$ ajustado (Adjusted R-squared) é 0,999I, o que indica que 99,9I\% de variação da variável resposta (X20I5) é explicada pelas variáveis de entrada. Quanto maior este valor melhor será o modelo porque irá explicar a maior parte da variabilidade observada na variável de resposta, a qual se busca prever.

Na Figura 8 é mostrado o diagnóstico do modelo de regressão e os gráficos dos valores residuais.

Figura 8. Diagnóstico do modelo.
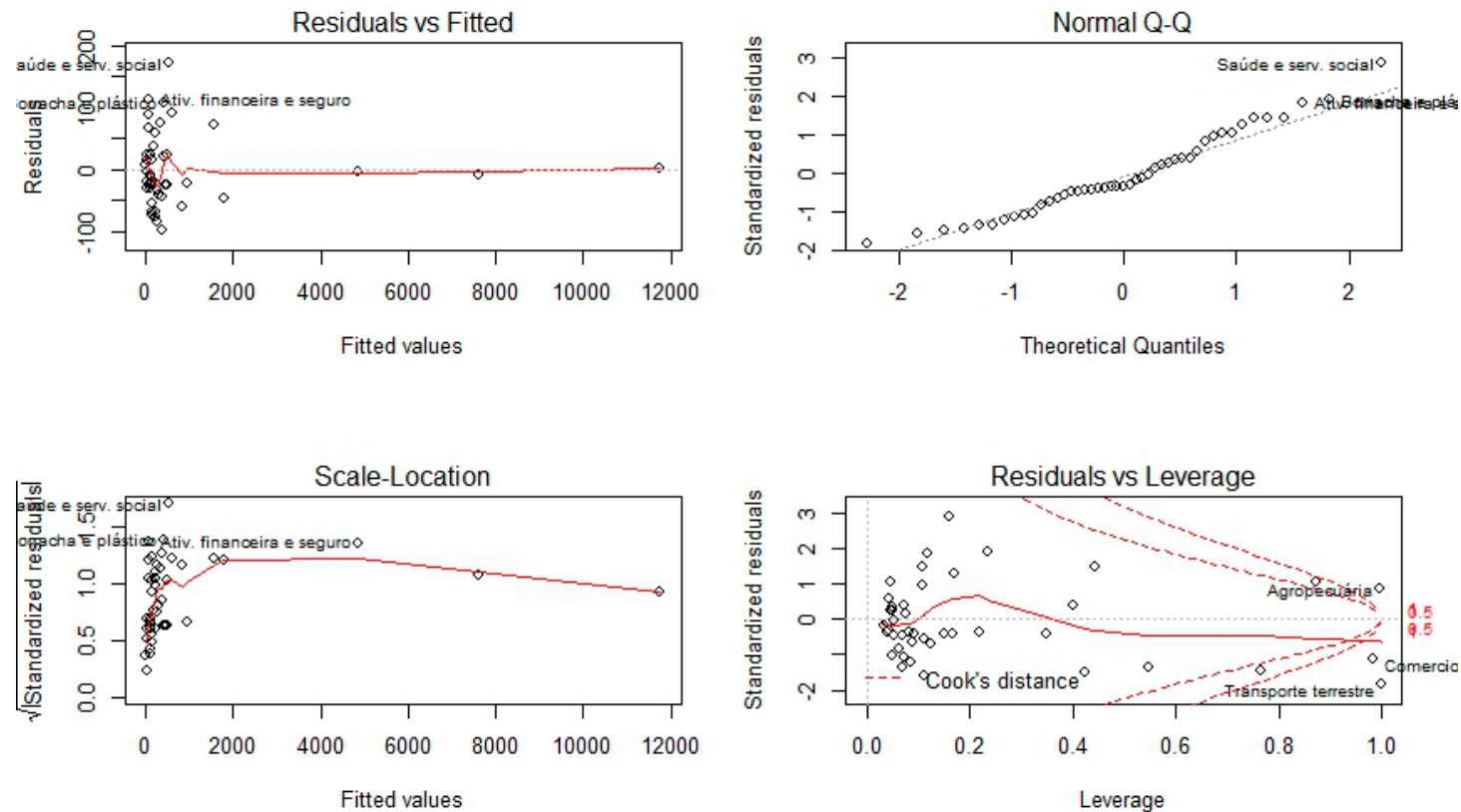

Pode-se fazer as seguintes observações a partir da plotagem dos valores nos gráficos mostrados na Figura 8:

- Os pontos em Residuals $\mathbf{x}$ Fitted parecem estar dispersos uniformemente no gráfico. Tais pontos verificam a homocedasticidade dos resíduos, a qual indica que a variância de erros é uniforme em praticamente todos os valores de $\mathrm{x}$;

- O gráfico Normal Q-Q contém pontos que se localizam em sua maioria na reta, indicando que os resíduos estão normalmente distribuídos;

- O gráfico Scale-Location mostra pontos que estão espalhadas em um padrão de largura constante, indicando variância constante;

- Existem também alguns pontos distintos de interesse contendo os nomes das áreas de negócios (outliers (pontos isolados) ou pontos de alavancagem) na plotagem.

Finalmente, na Figura 9 mostramos as Io primeiras áreas de negócios das pequenas e médias empresas e os valores previstos de desembolsos pelo BNDES para o ano seguinte aos utilizados no cálculo (2006 a 2015). 
Figura 9. Previsão calculada dos desembolsos.

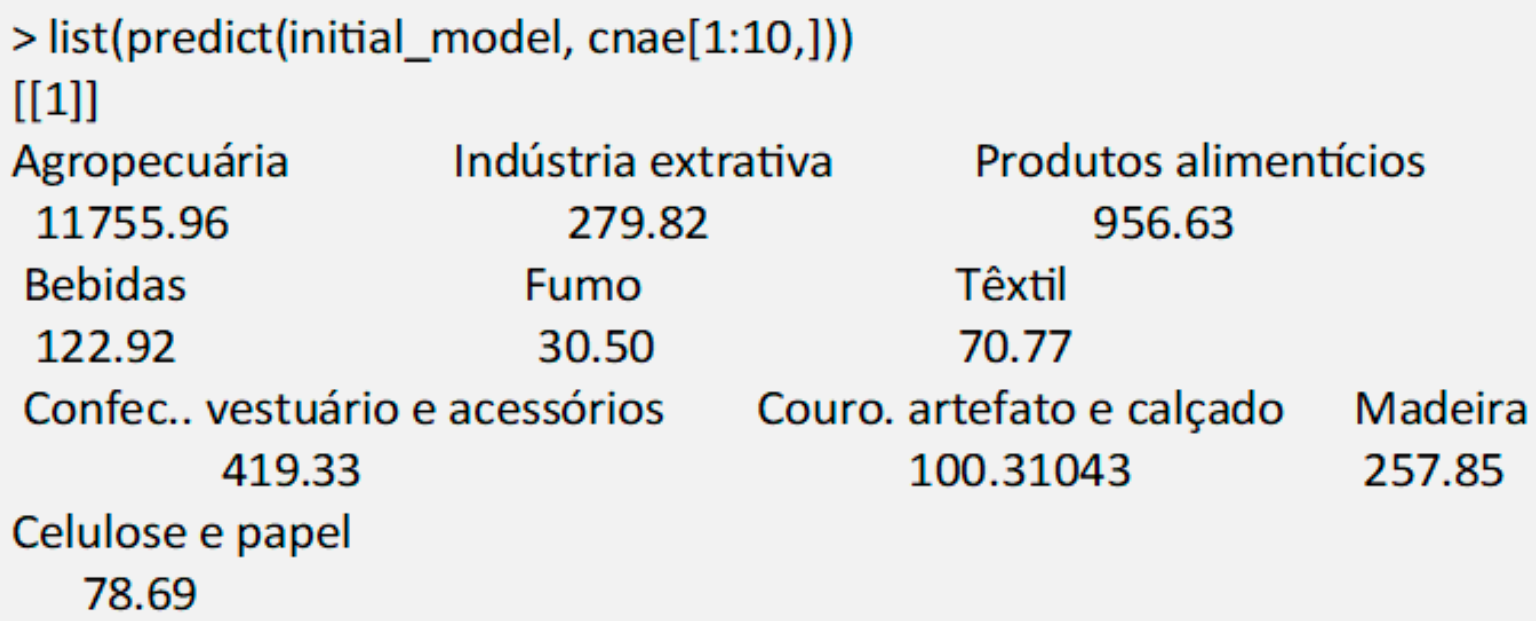

\section{RESULTADOS ESPERADOS E POSSÍVEIS APLICAÇÕES DO ESTUDO}

O trabalho teve como objetivo apresentar o ambiente de programação de código aberto $\mathrm{R}$ e seu ecossistema com o intuito de demonstrar a viabilidade do uso de tal ambiente pelas MPEs para que a análise de dados resultante possa ajudá-las em sua estratégia de inovação. Por ser um ambiente flexível e aberto, a linguagem $\mathrm{R}$ inclui em seu ecossistema inúmeros códigos prontos para uma ampla variedade de técnicas estatísticas o que facilita o seu aprendizado e utilização.

O artigo buscou também discutir e demonstrar uma abordagem sistemática (processo de ciência de dados) para a criação de aplicativos inteligentes de forma a que, utilizando elementos visuais como tabelas, gráficos e mapas, fornecer uma maneira acessível de extrair informações e descobrir padrões ocultos em dados "brutos".

Finalmente, buscou-se com este trabalho lançar as bases para a possibilidade de criar no País a sua própria comunidade de colaboradores no ecossistema R, o que sem dúvida, tornaria mais rápido o desenvolvimento de uma cultura científica de tratamento de dados abertos e suas ferramentas estatísticas e de modelagem.

\section{REFERÊNCIAS}

BECK, Thorsten. Financing Constraints of SMEs in Developing Countries: Evidence, Determinants and Solutions, Other publications TiSEM, Tilburg University, School of Economics and Management, AB Tilburg, Netherlands, 2007. Disponível em: https://EconPapers. repec.org/RePEc:tiu:tiutis:85aac075-08b5-44ce-bfia-96b2a50b64a6. Acesso em: 06 abr. 2016.

BIG DATA. Dicionário online do IATE - Europe Union Terminology [s.d.]. Disponível em: https://iate.europa.eu/entry/result/355I299/en-pt. Acesso em: II abr. 2016.

CAVIQUE, Luís - Big data e data science. Boletim da APDIO - Associação Portuguesa de Investigação Operacional, Lisboa, Portugal, n. 5I, 20I4, p. II-I4. 
DEJAEGER, Karel, et al. Beyond the Hype: Cloud Computing in Analytics, 20I2. Disponível em: http://dx.doi.org/I0.2I39/ssrn.2I65720. Acesso em: 07 abr. 2016.

DIAKOPOULOS, Nick, CASS, Stephen. Interactive: The Top Programming Languages 2015, IEEE Spectrum, New York, Estados Unidos, 26 jan. 20I6. Disponível em: https://spectrum. ieee.org/static/interactive-the-top-programming-languages-20I5. Acesso em: I7 jul. 2016.

GOVERNODIGITAL. Dados Abertos. [s.d.]. Disponível em: https://www.gov.br/governodigital/ pt-br/dados-abertos. Acesso em: I7 jul. 2016.

GOVERNO DIGITAL Dados Abertos Governamentais. 05 dez. 20I5. Disponível em: https:// www.gov.br/governodigital/pt-br/dados-abertos/dados-abertos-governamentais. Acesso em: I7 jul. 2016.

ISOTANI, Seiji; BITTENCOURT, Ig Ibert. Dados Abertos Conectados. São Paulo: Novatec, 20I5. I76 páginas. Disponível em:http://ceweb.br/publicacao/livro-dados-abertos. Acesso em: 07 abr. 2016.

IWASAKI, Eliane Yumi. Movimento Open Source: A Importância da Comunicação e da Relação Entre Empresas e Comunidades para o Mercado. 2008. 4I F. Trabalho de Conclusão de Curso (Especialização)-Faculdade de Comunicação Social Cásper Libero. São Paulo, 2008. KABACOFF, Robert. $\mathbf{R}$ in Action: Data analysis and graphics with $\mathbf{R}$, Second Edition, Manning Publications, New York, ESTADOS UNIDOS, 2015. p. 608.

KRILL, Paul. Why R? The Pros and Cons of the $\mathbf{R}$ Language, InfoWorld, IDG Communications, Massachusetts, Estados Unidos, 30 jun. 20I5. Disponível em: https://www. infoworld.com/article/2940864/r-programming-language-statistical-data-analysis.html. Acesso em: 08 abr. 2016.

LEME, Luisa. São Paulo 20I4 Blog: Six Facts about SMEs in Brazil, Americas Society Council of the Americas, Nova York, Estados Unidos, I2 mar. 20I4. Disponível em: https://www.as-coa. org $/$ blogs $/ \mathbf{s} \% \mathrm{C}_{3} \% \mathrm{~A}_{3} 0$-paulo-20I4-blog-six-facts-about-smes-brazil. Acesso em: 08 abr. 2016.

MANOCHA, Ian. On the Road to Open Data, IDG Connect, Londres, Reino Unido, o8 Ago. 20II. Disponível em: https://www.idgconnect.com/idgconnect/opinion/I02I406/ian-manochauk-road. Acesso em: 08 abr. 2016.

MARKOFF, John. Entrepreneurs See a Web Guided by Common Sense, The New York Times, I2 nov. 2006. Disponível em: https://www.nytimes.com/2006/II/I2/business/I2web.html. Acesso em: 07 abr. 2016.

MITCHELL, Melaine; TURNER, Jamie; CORBETT, R. Scott; TASNER, Michael. Measure the Impact of Online Marketing (Collection), FT Press, New Jersey, Estados Unidos, $20 \mathrm{I} 2$. p.329.

PREEZ, Dereck Du. Big Data for Small Business, Raconteur Media Ltd., London, Reino Unido, 9 set. 20I4. Disponível em: https://www.raconteur.net/technology/big-data-for-small- 
business. Acesso em: 6 abr. 2016.

R CORE TEAM. R: A language and environment for statistical computing. $R$ Foundation for Statistical Computing, Vienna, Austria, 2013. Disponível em: http://www.R-project.org/. Acesso em: 07 abr. 2016.

RIBEIRO, Claudio Jose Silva; ALMEIDA, Reinaldo Figueiredo. Dados abertos governamentais (Open Government Data): instrumento para exercício de cidadania pela sociedade. Anais do XII Encontro Nacional de Pesquisa em Ciência da Informação Políticas de Informação para a Sociedade, Brasília, 23 a 26 de out. 20II. Disponível em: http://arq.3rengtt.com.br/wp-content/ uploads/20I5/09/EnancibXII-RibeiroAlmeida.pdf. Acesso em: 07 abr. 2016.

RIJMENAM, Mark van. Also SMEs Can Achieve Remarkable Results with Big Data, Datafloq, Hague, Netherlands, 23 mai. 2015. Disponível em: https://datafloq.com/read/alsosmes-can-achieve-remarkable-results-with-big-/192. Acesso em: 06 abr. 2016.

SAS INSTITUTE. SAS Overview and Annual Report, SAS Institute Inc., North Caroline, Estados Unidos, 20I3. p.I04.

SCHUTT, Rachel; O'NEIL, Cathy. Doing Data Science: Straight Talk from the Frontline, O'Reilly Media, California, Estados Unidos, 20I3. p. 375.

SIMON, Phil. Too Big to Ignore: The Business Case for Big Data, John Wiley \& Sons, New Jersey, Estados Unidos, 2015. p.256.

SMITH, David. Why now is the time to learn R?, Open source.com - Articles, North Caroline, Estados Unidos, 27 jan. 2015. Disponível em: https://opensource.com/business/14/12/r-opensource-language-data-science. Acesso em: 06 abr. 2016.

THE ECONOMIST. Data, data everywhere, The Economist - Special Edition, London, Reino Unido, 27 fev. 20Io. Disponível em: https://www.economist.com/special-report/20Io/02/27/datadata-everywhere. Acesso em: 2I jun. 2016.

THE R PROJECT, R: A Language and Environment for Statistical Computing. R Foundation for Statistical Computing, Vienna, Austria, 2015.

UBALDI, Barbara. Open Government Data: Towards Empirical Analysis of Open Government Data Initiatives, OECD Working Papers on Public Governance, No. 22, OECD Publishing, Paris, 27 mai. 20I3. Disponível em: https://doi.org/I0.I787/5k46bj4fo3s7-en. Acesso em: I6 out. 2015 . 